\title{
Phase separation phenomena in solutions of polysulfone in mixtures of a solvent and a nonsolvent: relationship with membrane formation*
}

\author{
J. G. Wijmans, J. Kant, M. H. V. Mulder and C. A. Smolders \\ Department of Chemical Technology, Twente University of Technology, PO Box 217, \\ 7500 AE Enschede, The Netherlands \\ (Received 22 October 1984)
}

\begin{abstract}
The phase separation phenomena in ternary solutions of polysulfone (PSf in mixtures of a solvent and a nonsolvent ( $N, N$-dimethylacetamide (DMAc) and water, in most cases) are investigated. The liquid-liquid demixing gap is determined and it is shown that its location in the ternary phase diagram is mainly determined by the PSf-nonsolvent interaction parameter. The critical point in the PSf/DMAc/water system lies at a high polymer concentration of about $8 \%$ by weight. Calorimetric measurements with very concentrated PSf/DMAc/water solutions (prepared through liquid-liquid demixing, polymer concentration of the polymer-rich phase up to $60 \%$ ) showed no heat effects in the temperature range of $-20^{\circ} \mathrm{C}$ to $50^{\circ} \mathrm{C}$. It is suggested that gelation in PSf systems is completely amorphous. The results are incorporated into a discussion of the formation of polysulfone membranes.
\end{abstract}

(Keywords: polysulfone; solutions; liquid-liquid demixing; crystallization; membrane structures; membrane formation)

\section{INTRODUCTION}

The field of membrane filtration covers a broad range of different separation techniques such as: hyperfiltration, reverse osmosis, ultrafiltration, microfiltration, gas separation and pervaporation. Each process makes use of specific membranes which must be suited for the desired separation. This implies that many different membranes with optimized properties have to be made and consequently it means that there is a strong interest among membrane manufacturers in the parameters that govern these membrane properties.

In our laboratory we try to obtain a coherent view on the formation of membranes. In this mechanism of formation an important role is played by the phase transition phenomena (gelation and liquid-liquid demixing) in ternary polymer solutions. An introduction to the formation mechanism will be given in the theory section.

The phase separation phenomena in the membrane forming system consisting of cellulose acetate, dioxan and water have been studied by Altena ${ }^{1}$. In the present work we will focus on systems which contain the polymer polysulfone. Polysulfone is an important polymer in commercial membrane fabrication, especially as an ultrafiltration membrane and as a support for composite membranes. The system studied most thoroughly consists of polysulfone, $N, N$-dimethylacetamide (a solvent) and water (a nonsolvent). We will determine the liquid-liquid demixing gap and we will investigate whether the transition from a fluid to a solid state (gelation) in this system is accompanied by crystallization.

\footnotetext{
*A part of this work was presented at the Fourth Symposium on Synthetic Membranes in Science and Industry, Tübingen, FRG, 6-9 September 1983
}

\section{THEORY}

\section{Membrane formation}

In the phase inversion process a membrane is made by casting a polymer solution on a support and then bringing the solution to phase separation by means of solvent outflow and/or nonsolvent inflow. Thus, in most cases at least three components are involved: a polymer, a solvent and a nonsolvent. Exchange of the latter two leads to a phase transition in the at first homogeneous polymer solution and the membrane structure is formed. The cross section of this structure is asymmetric in many cases: a thin and dense skin layer is supported by a porous sublayer. In our view two different types of phase separation are responsible for these two layers ${ }^{1-5}$ :

-gelation (possibly induced by crystallization) for the formation of the skin layer;

-liquid-liquid phase separation followed by gelation of the concentrated phase for the formation of the porous sublayer.

Gelation will take place at high polymer concentrations whereas liquid-liquid phase separation is expected to occur at low polymer concentrations. In Figure $l$ the phase boundaries are schematically drawn in a ternary phase diagram. The arrows 1 and 2 represent the change in composition for respectively the skin layer and the sublayer if a polymer film with initial composition $A$ is immersed in a nonsolvent bath. In this approach it is assumed that the ratio of solvent outflow to nonsolvent inflow is larger for the skin layer (in direct contact with the coagulation bath) than for the sublayer.

\section{Liquid-liquid phase separation}

Altena $^{6}$ has shown that in virtually every system consisting of a polymer, a solvent and a nonsolvent 


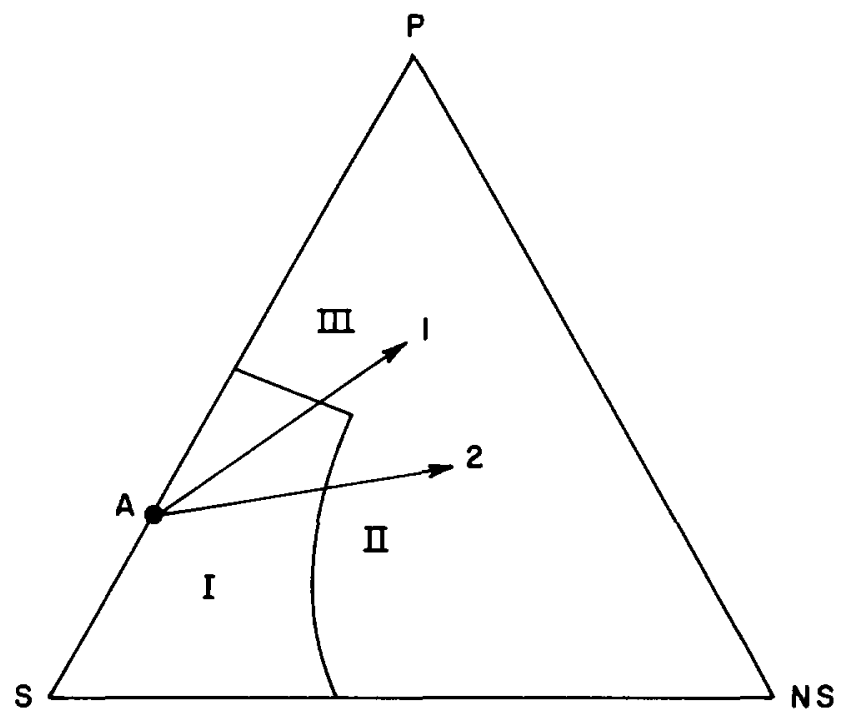

Figure 1 Schematic representation of a ternary phase diagram. I: homogeneous solution; II: liquid-liquid demixing; III: gelation. The arrows are possible coagulation paths, see text

liquid-liquid demixing can be expected. The exact location of the demixing gap is determined by the interactions between the three components. The liquidliquid phase boundary is the so-called binodal. Every composition inside the binodal will demix into two liquid phases which differ in compositions but which are in thermodynamic equilibrium with each other. The line which connects a pair of equilibrium compositions in the phase diagram is called a tie line, see Figure 2. The two compositions are identical at the critical point. Liquidliquid demixing is reasonably well described by FloryHuggins thermodynamics ${ }^{7}$ for ternary systems. In this description the interactions between the components are represented by three binary interaction parameters: $\chi_{\text {polymer solvent }}, \chi_{\text {solvent nonsolvent }}$ and $\chi_{\text {polymer, nonsolvent }}$. These interaction parameters incorporate both enthalpy and entropy contributions.

There are three different ways for liquid-liquid demixing to set in: (i) by nucleation and growth of the concentrated phase (rich in polymer), (ii) by nucleation and (iii) by spinodal decomposition. The second phenomenon is the most important in membrane formation. It leads to a continuous polymer matrix in which spheres filled with the diluted phase (in general with an extremely low polymer content) are dispersed. For an effective membrane the dilute phase forms also a continuous phase (by coalescence of the spheres).

\section{Gelation}

At high polymer concentrations polymer solutions are able to form a three dimensional network and in that case the fluid system is transformed into a gel. The nature of the crosslinks which form the network may differ from system to system ${ }^{8}$. For crystalline or semicrystalline polymers the crosslinks are micro-crystallites and the thermoreversible gelation can be described by the theory of melting point depression ${ }^{7}$. Gelation in the membrane forming system cellulose acetate/dioxan/water has been analysed in this way ${ }^{1}$.

Recently attention has been paid to gelation phenomena in solutions of noncrystalline polymers by Tan et $a .^{9,10}$. Both in solutions of atactic polystyrenes ${ }^{9}$ and of some chlorinated polyethylenes ${ }^{10}$ thermoreversible gelation is observed. The melting of these gels is even accompanied by a very small endothermic peak in d.s.c. experiments. These findings raise interesting questions regarding the nature of the interchain connections in gels of amorphous polymers and these are addressed in the papers by Tan et al. They suggest that chain overlap is a necessary condition for gel formation and that the network junctions are stabilized through local ordering. The physical picture of the latter process is not clear at the moment.

\section{EXPERIMENTAL}

\section{Materials}

The polymer polysulfone P3500 (PSf) was purchased from Union Carbide. It is characterized by $M_{\mathrm{n}}=14000$, $M_{\mathrm{w}}=46000$ and $M_{z}=75000$ (determined by h.p.l.c.). The solvents and nonsolvents used were of reagent grade and were used without further purification, except for drying on molecular sieves.

\section{Cloud points}

The cloud points in ternary systems are determined in two ways: (1) by a rapid titration method and (2) by turbidity measurements upon cooling a ternary solution. The second method is the more accurate one.

(1) A solution of the polymer in the solvent is placed in a vessel which is kept at a constant temperature $\left(25^{\circ} \mathrm{C}\right.$ normally). To this solution the pure nonsolvent or a mixture of the solvent and the nonsolvent is slowly added, see Figure 3. This is continued until permanent turbidity is detected visually. The composition of the solution in the vessel at the moment is computed from the total amounts of polymer, solvent and nonsolvent present. The addition of mixtures containing a considerable fraction of solvent is favoured over the addition of pure nonsolvent since in the latter case every drop added will cause local coagulation in the polymer solution. To allow the solution to become homogeneous again may take a long time.

(2) A method described earlier by van Emmerik ${ }^{11}$ has been modified to make the procedure less laborious. The experimental setup is shown in Figure 4. The temperature of the thermostat bath is decreased by an external

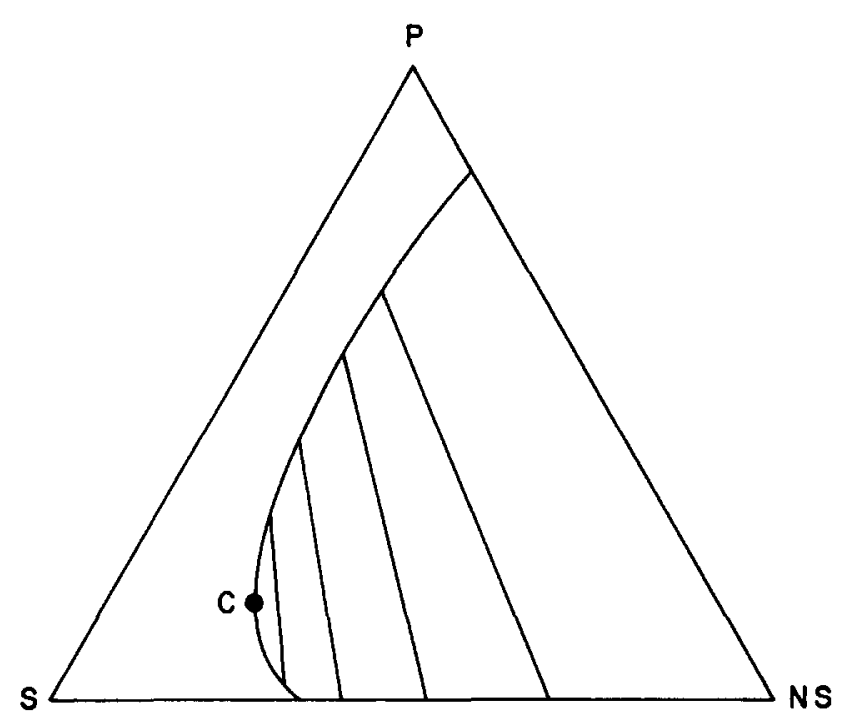

Figure 2 Liquid-liquid demixing gap in a ternary phase diagram. The straight lines in the demixing gap are tie lines. $C$ is the critical point 


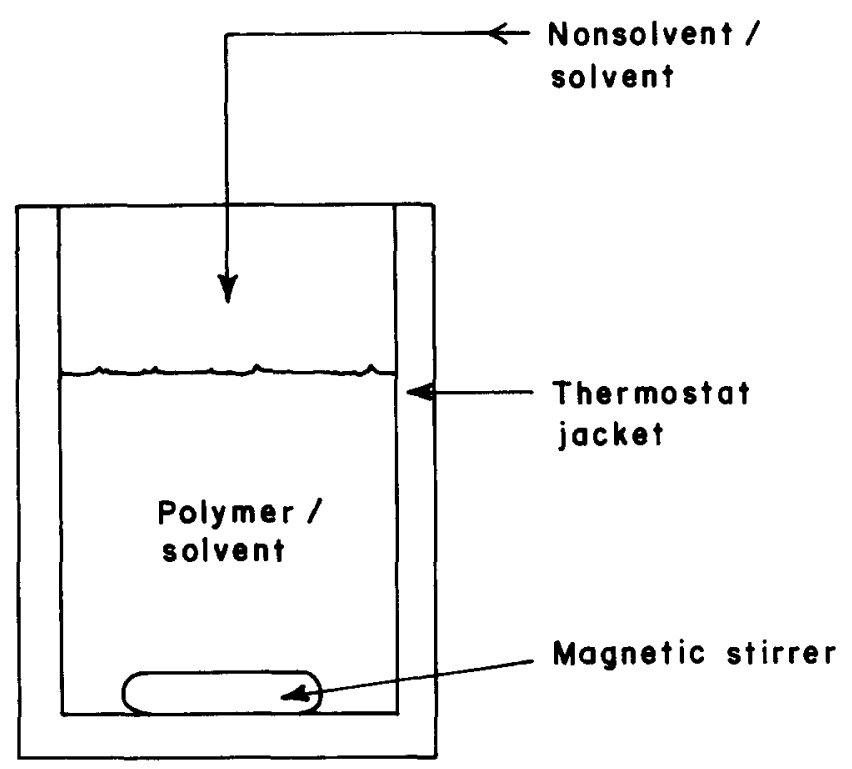

Figure 3 Determination of cloud points by the titration method

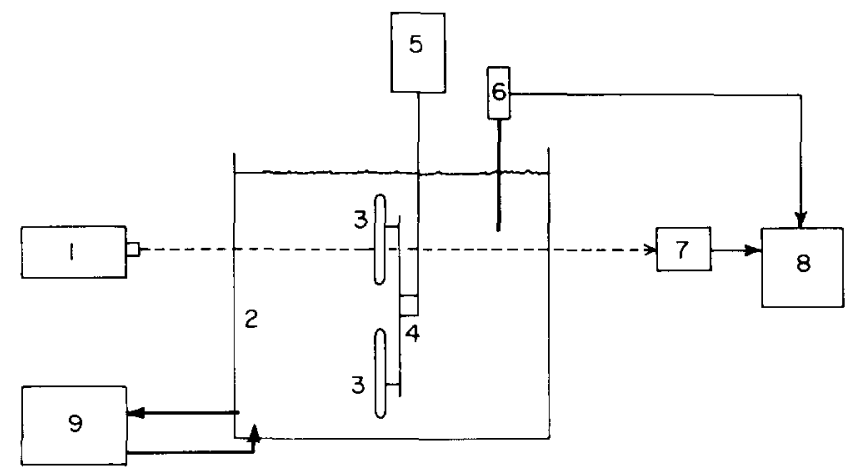

Figure 4 Detection of cloud point temperature by turbidity measurements: 1 , laser; 2 , thermostat bath; 3 , capillary tubes; 4 , rotating wheel; 5 , motor; 6 , thermistor; 7 , detector; 8 , recorder; 9 , cryostat

cooling unit at a constant rate of $1^{\circ} \mathrm{C}$ per $10 \mathrm{~min}$. In the bath a slowly rotating wheel is placed, to which a number of capillary tubes is attached. Each tube is filled with a polymer/solvent/nonsolvent mixture of a certain composition. The tubes have been sealed under vacuum at liquid nitrogen temperature. Due to the rotation of the wheel each tube intersects the laser beam every $5 \mathrm{~min}$. The intensity of the transmitted laser light is continuously monitored on a recorder together with the temperature of the bath. The at first homogeneous solutions in the tubes will demix as a result of the decrease in temperature and this is observed in the recorder output as illustrated in Figure 5. Part (a) of this Figure displays the recorder output. If there is no tube between the laser and the detector the transmission is $100 \%$. At the moment the wall of a tube intersects the laser beam, all the light is reflected and the transmission is zero. Moments later the laser beam passes through the centre of the tube and if the solution is clear the transmission is $100 \%$ or a little bit less. The transmission is zero again at the moment the tube leaves the beam. After a short time the next tube appears, etc. In part (b) it is shown how the cloud point temperature of a certain tube is determined by interpolation. The compositions in the tubes belong to a homologous series. These series can be made (i) by adding different amounts of polymer to a certain solvent/nonsolvent mixture (see
Figure $6 a$ ), or (ii) by mixing a solution of the polymer in the solvent in different ratios with a solvent/nonsolvent mixture, see Figure $6 b$. For each series the cloud point temperatures are plotted as a function of the polymer concentration and through interpolation the compositions which demix at a certain temperature $\left(25^{\circ} \mathrm{C}\right.$ normally) are determined. The choice of the concentration range to be examined is facilitated if some titration experiments as described above are performed previously.

\section{Tie lines}

If the polymer/solvent/nonsolvent mixture in a tube has been subjected to liquid-liquid phase separation, it is in principle possible to separate the two equilibrium phases from each other by centrifugation. Problems arise if gelation of the concentrated phase occurs. This is not the case in PSf systems and centrifugation $(2000 \mathrm{rpm})$ yields two completely clear phases with a distinct interface. The more viscous the concentrated phase the more tedious this procedure will be. The tube is then placed vertically with the concentrated phase on top. The diluted phase flows to the bottom of the tube, whereas the concentrated phase remains in the upper part. Upon breaking the tube, a sample can be taken from both phases and the polymer concentrations can be determined by evaporation of the solvent and the nonsolvent.

\section{Differential scanning calorimetry}

The apparatus used was a Perkin Elmer DSC model II, equipped with an external cooling unit (liquid nitrogen) to make experiments possible at subambient temperatures. The d.s.c. samples can be prepared in two different ways.

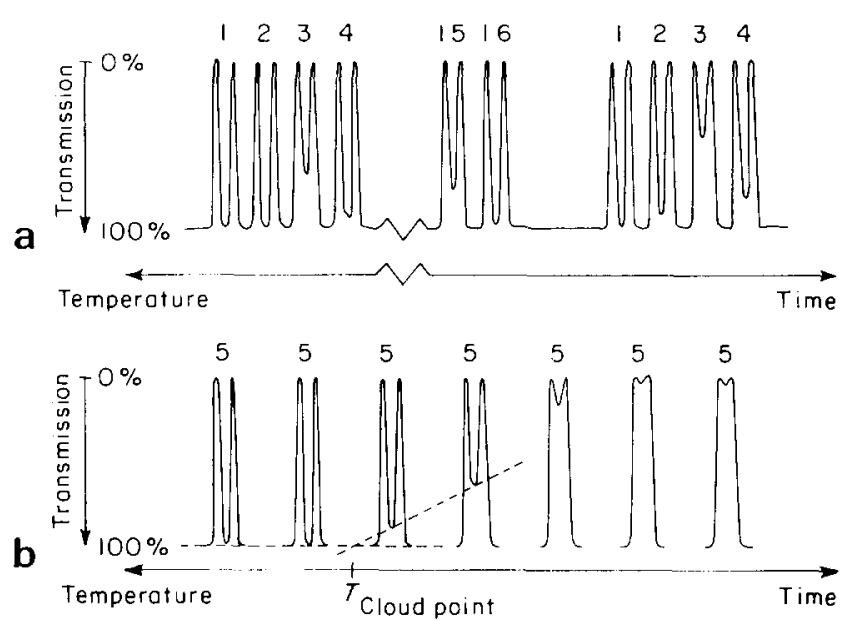

Figure 5 (a) Trace of recorder output of turbidity experiment. Numbers identify separate tubes. (b) Determination of the cloud point temperature of tube no. 5 through interpolation

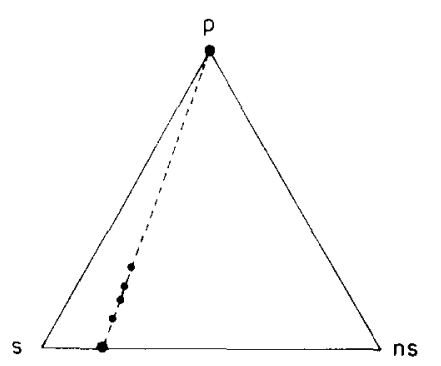

a

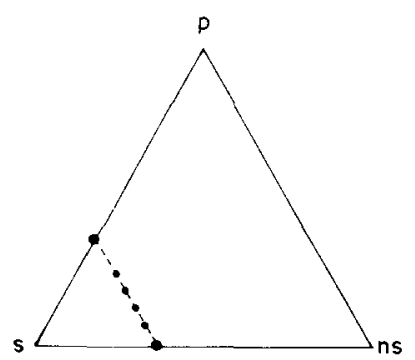

b
Figure 6 Pseudo-binary series of compositions 


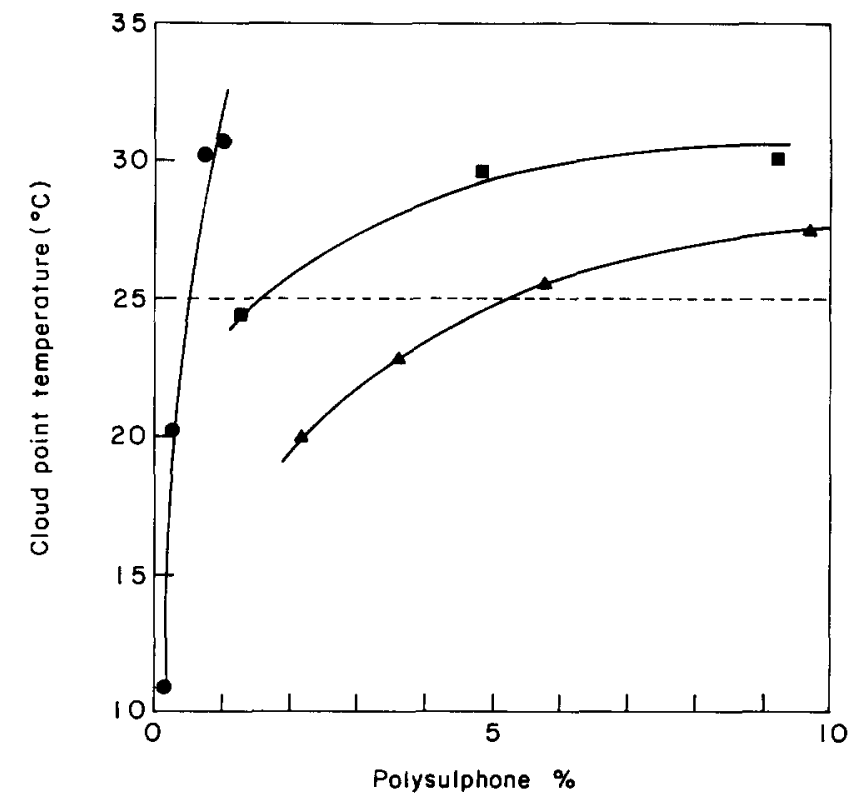

Figure 7 Cloud point temperatures of three pseudo-binary series. ( $\mathrm{H}_{2} \mathrm{O} / \mathrm{DMAc}$ ratio $=4.42 / 95.58 ;(\square) \mathrm{H}_{2} \mathrm{O} / \mathrm{DMAc}$ ratio $=4.27 / 95.73 ;(\Delta)$ $\mathrm{H}_{2} \mathrm{O} / \mathrm{DMAc}$ ratio $=4.22 / 95.78$

(1) The appropriate amounts of PSf (powder) and solvent/nonsolvent mixture are weighed into the aluminium sample pans. The PSf granules were converted into a powder as described recently ${ }^{12}$. The pans are sealed and are stored at a temperature of 90 or $120^{\circ} \mathrm{C}$ for several days. After this period the sample is weighed again and only sample pans that showed no weight loss are used in the experiments. For the present purpose of the d.s.c. experiments, this preparation method has the following disadvantages: (i) PSf concentrations higher than $35 \%$ by weight are difficult to obtain, and (ii) there is no direct proof that the samples have become completely homogeneous during the waiting period at elevated temperatures, molecular diffusion being the sole mixing mechanism.

(2) During the determination of the tie lines, it became clear that the concentrated phase of liquid-liquid demixed systems had high, to very high, PSf concentrations. This has been used to prepare concentrated samples for the d.s.c. experiments.

As in the determination of the tie lines, the concentrated phase of a demixed PSf/DMAc/water is isolated. From this phase a small amount $(\sim 10 \mathrm{mg})$ is put in a sample pan and another part is used to determine the PSf concentration. In this way it is possible to prepare samples with PSf concentrations up to $60 \%$ by weight of which it is certain that they are homogeneous at room temperature. All d.s.c. experiments presented in this paper have been carried out with samples prepared by method 2 .

The samples are cooled from $50^{\circ} \mathrm{C}$ to $-20^{\circ} \mathrm{C}$ or $-70^{\circ} \mathrm{C}$ at a rate of $10^{\circ} \mathrm{C} \mathrm{min}{ }^{-1}$. After a waiting period at the lower temperature (varying from 0 to $20 \mathrm{~h}$ ) the sample is reheated to $50^{\circ} \mathrm{C}$ at a rate of $10^{\circ} \mathrm{C} \mathrm{min}^{-1}$. During the heating run the d.s.c. signal is recorded in order to detect heat effects.

\section{RESULTS AND DISCUSSION}

\section{Polysulfone/solvent mixtures}

The solvent DMAc is a good solvent for polysulfone, so it is expected that binary PSf/DMAc mixtures are thermo- dynamically stable up to high PSf concentrations. Contrary to this is the fact that solutions of PSf in DMAc and in other solvents become turbid after a certain time and that a white precipitate is formed. In a recent publication $^{12}$ we have shown that this instability is due to crystallization of a small oligomer fraction of the polysulfone. Once this fraction is removed, the solutions are stable for $>6$ months at least. In solutions of PSf in DMAc this oligomer crystallization does not set in within one day if the PSf concentration is lower than $15 \%$.

\section{Cloud points}

The cloud point temperatures of homologous series of PSf/DMAc/water mixtures have been determined by the turbidity method. In Figure 7 the demixing temperatures are displayed for three different series. Through interpolation the compositions which demix at $25^{\circ} \mathrm{C}$ are obtained and these compositions are shown in the phase diagram of Figure 8. The water concentration varies from $3.0 \%$ to $4.2 \%$ when the PSf concentration goes from $25 \%$ to $1 \%$. In this range the cloud points lie on a straight line with very little deviation. At PSf concentrations lower than $1 \%$ the binodal bends sharply towards higher water concentrations and vanishes in the water-DMAc axis for water concentrations larger than $5 \%$. The six cloud points

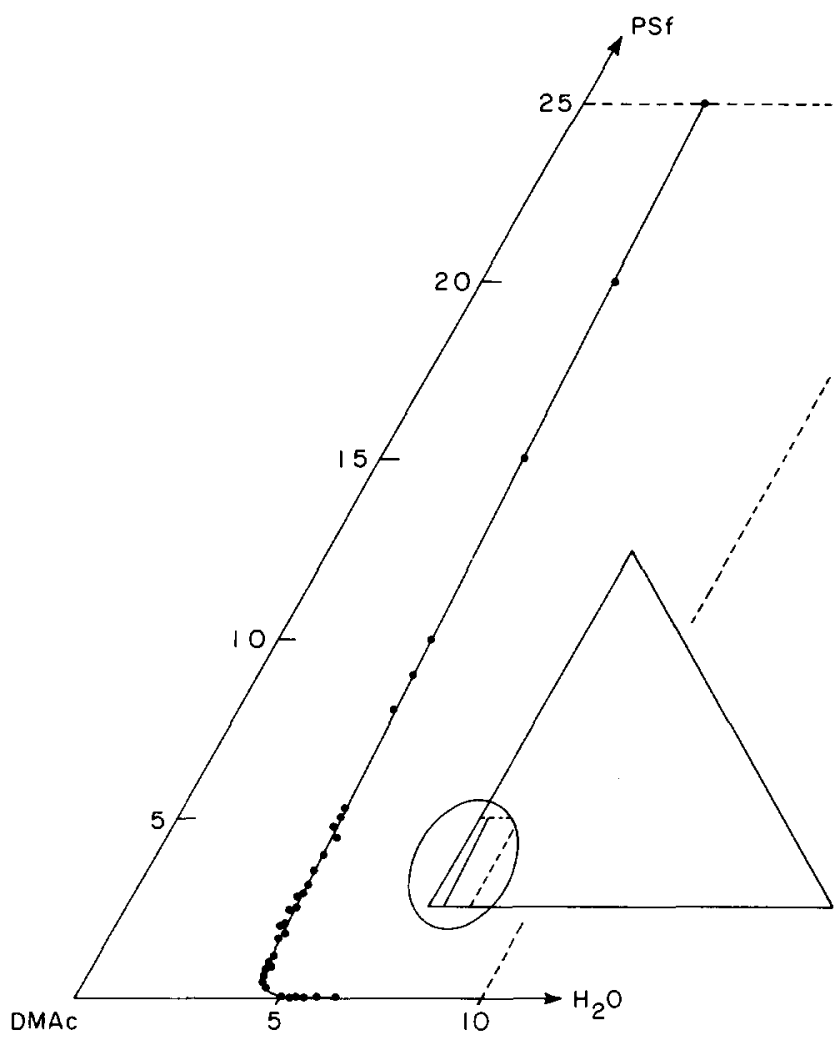

Figure 8 Cloud points in the system consisting of polysulfone, DMAc and water at $25^{\circ} \mathrm{C}$

Table 1 Cloud points at low PSf concentrations

\begin{tabular}{lll}
\hline$\%$ PSf & $\% \mathrm{H}_{2} \mathrm{O}$ & $\%$ DMAc \\
\hline 0.042 & 5.10 & 94.86 \\
0.024 & 5.30 & 94.68 \\
0.015 & 5.50 & 94.49 \\
0.0095 & 5.70 & 94.29 \\
0.0060 & 6.00 & 93.99 \\
0.0018 & 6.45 & 93.55 \\
\hline
\end{tabular}




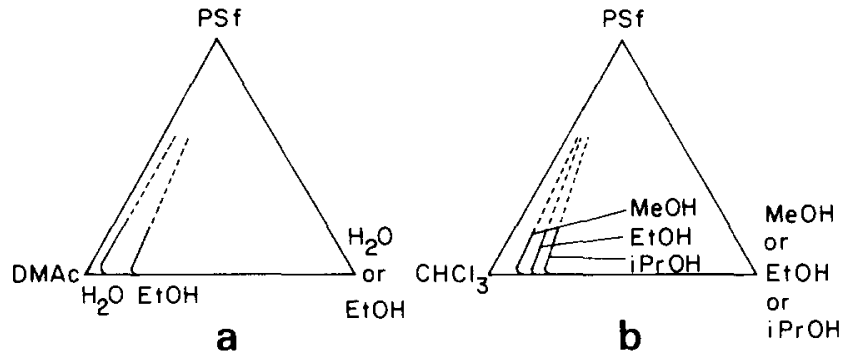

Figure 9 Binodals in five different systems with polysulfone and varying solvents and nonsolvents. $T=25^{\circ} \mathrm{C}$

with very low PSf concentrations have been determined by the titration method and the compositions are given in Table 1. At such low polymer concentrations the detection of the cloud point is not very accurate and the values of Table 1 are the mean values of in total 24 experiments.

Polysulfone is a very hydrophobic polymer, so the PSfwater interaction parameter will have a large positive value. This parameter has been determined by swelling experiments and a value of 5.9 was obtained ${ }^{13}$. The theoretical phase diagrams, as calculated by Altena ${ }^{6}$ for ternary systems with a large polymer-nonsolvent interaction parameter, display a binodal located close to the polymer-solvent axis, regardless of the choice of the solvent. The experimentally determined binodal is in agreement with this observation.

Next to the PSf/DMAc/water system the binodals in other polysulfone systems have been determined by the titration method. The binodals are shown in Figure 9 (a: PSf/DMAc/water or ethanol, and b: $\mathrm{PSf} / \mathrm{CHCl}_{3} /$ methanol or ethanol or iso-propanol). Figure 9 has previously been published by Mulder et al. ${ }^{14}$ in their study of the preparation of asymmetric pervaporation membranes. The binodals of the systems PSf/D$\mathrm{MAc} / \mathrm{EtOH}$ and $\mathrm{PSf} / \mathrm{CHCl}_{3} / \mathrm{EtOH}$ are almost identical. This once again shows that the polymer-nonsolvent interaction is very important in these systems. In the homologous series of nonsolvents, the binodal shifts to higher nonsolvent concentrations going from water to isopropanol. In the same series the polysulfone-nonsolvent repulsion, and thus $\chi_{\mathrm{PSf} \text { nonsolvent, decreases }}$ $\left(\chi_{\mathrm{PSP} \text { EIOH }}=2.5^{13}\right)$. From these observations it is clear that in ternary systems with strong polymer-nonsolvent repulsions the value of $\chi_{\text {polymer, nonsolvent }}$ determines to a large extent the location of the liquid-liquid demixing gap.

\section{Tie lines}

The determination of the polymer concentration in the concentrated and dilute phases is not very accurate. This is illustrated by the fact that an increase in the PSf concentration in the concentrated phase is not always accompanied by a decrease in the PSf concentration in the dilute phase. In a truly ternary system this should be the case. The presence of a polymer component makes our system a pseudo-ternary system, but at present we feel that the molecular weight distribution of the polymer is not very relevant. The uncertainty in the experiments is probably due to the fact that during the centrifugation step no temperature control was possible.

In Figure 10 the PSf concentration in the concentrated phase is plotted as a function of the PSf concentration in the dilute phase for five different experiments. The points above and under the diagonal are identical. Figure 10 shows (i) that the PSf concentrations in the concentrated phase are very high, up to $60 \%$, (ii) that the tie lines are extremely steep, i.e. they are almost parallel to the PSfDMAc axis, and (iii) that the critical point, where the two concentrations are identical, probably lies at a PSf concentration around $8 \%$.

In the first part of this section, attention has been paid to the oligomer crystallization in concentrated PSf solutions. The concentrated phase of a liquid-liquid demixed system does not show this behaviour, since the oligomer fraction accumulates in the PSf-poor phase ${ }^{12}$.

\section{Gelation}

Samples with PSf concentrations ranging from $40 \%$ to $60 \%$ have been examined. Due to the preparation method these samples are known to be fluid at $25^{\circ} \mathrm{C}$ and they contain a small percentage of water. Extrapolation of the binodal to high PSf concentrations suggests that the water concentration is about $2 \%$.

All samples were cooled from $50^{\circ} \mathrm{C}$ to $-70^{\circ} \mathrm{C}$ and then immediately reheated to $50^{\circ} \mathrm{C}$. In the heating run melting endotherms were detected in the range of $-40^{\circ} \mathrm{C}$ to $-25^{\circ} \mathrm{C}$,

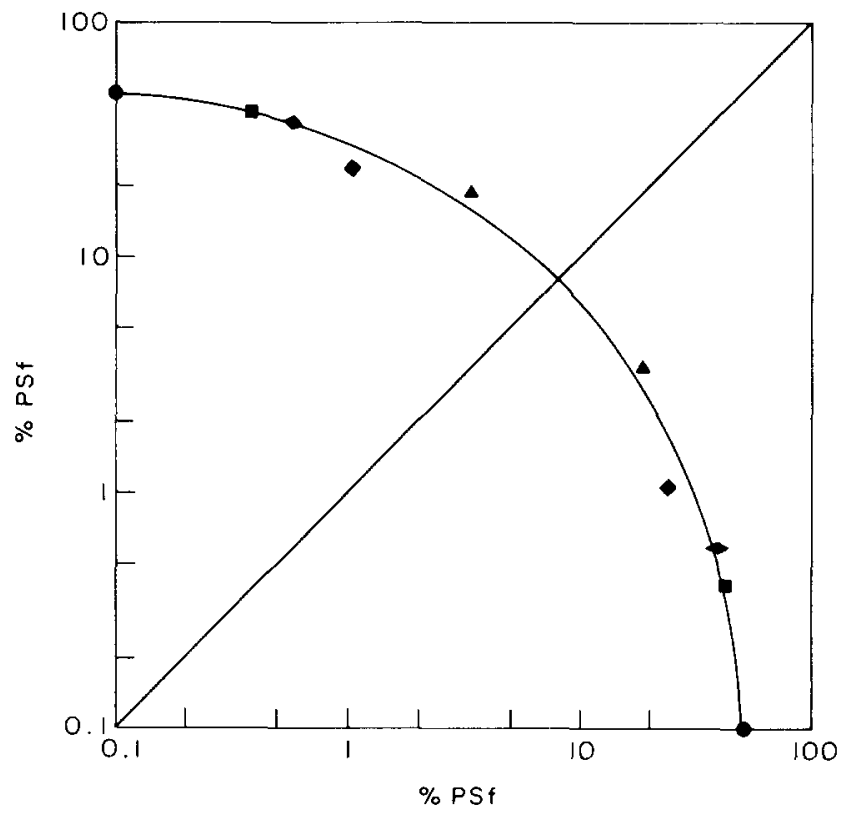

Figure 10 PSf concentration in the concentrated phase as a function of the PSf concentration in the corresponding dilute phase. The points on either side of the diagonal are identical

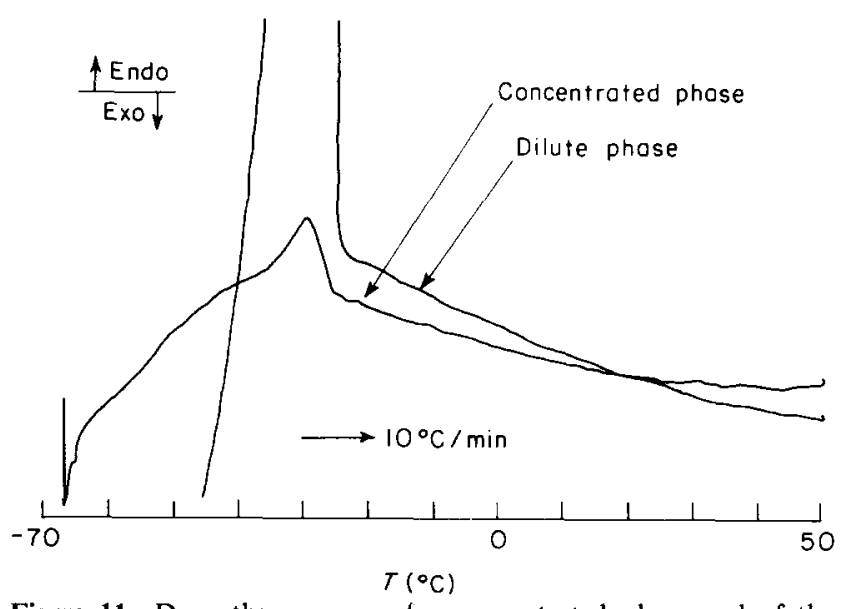

Figure 11 D.s.c. thermogram of a concentrated phase and of the corresponding dilute phase. The PSf concentration in the concentrated phase is about $60 \%$ 


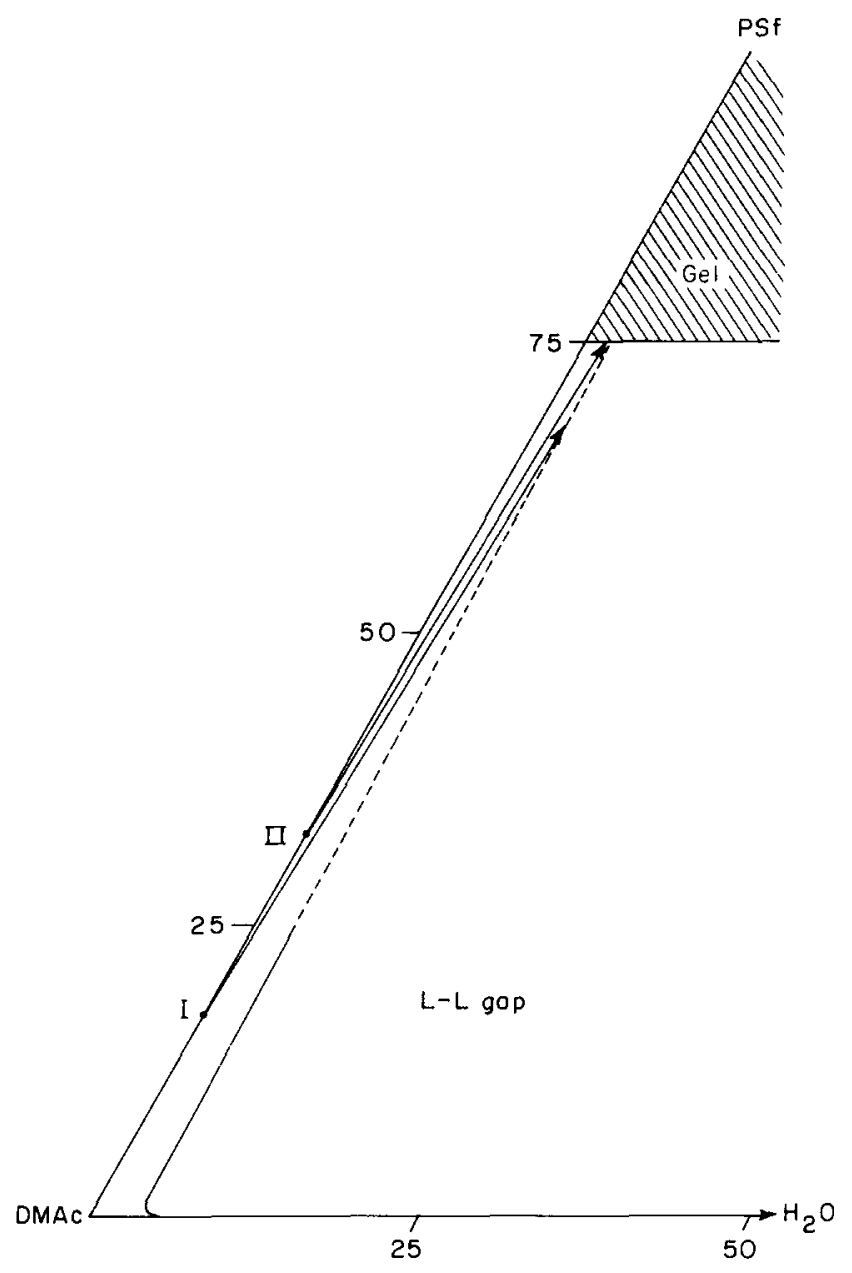

Figure 12 Ternary phase diagram. The gel region is arbitrarily defined to start at a PSf concentration equal to $75 \%$. The binodal is extrapolated from Figure 8 for PSf concentrations higher than $25 \%$. I and II indicate the initial composition of the casting solution in the membrane-forming systems I and II (see Table 2). The coagulation paths drawn in the phase diagram are possible paths; they have not been calculated or measured

see Figure 11. The melting peak is larger and sharper than expected for the melting of a polymer and this raises the question whether low molecular weight components are responsible for this transition energy. That this indeed is the case is proven by the following experiment: the corresponding dilute phase is cooled to $-70^{\circ} \mathrm{C}$ and then reheated to $50^{\circ} \mathrm{C}$. A very large melting effect is observed in the same temperature range as the endotherm of the concentrated sample. The fact that the melting effect is larger for the polymer-poor phase indicates that not the polysulfone but the DMAc/water mixture crystallizes. This conclusion is supported by the observation that the melting point of a binary DMAc/water mixture with a DMAc concentration of $95 \%$ is about $-30^{\circ} \mathrm{C}$. The two peaks in Figure 11 coincide in temperature because the two samples are in thermodynamic equilibrium with each other.

In order to avoid the crystallization of the solvent and the nonsolvent the lower temperature boundary was set at $-20^{\circ} \mathrm{C}$ in the next series of experiments. The samples were cooled to $-20^{\circ} \mathrm{C}$ and reheated to $50^{\circ} \mathrm{C}$ after a waiting period of 0 to $20 \mathrm{~h}$. In none of the heating runs heat effects could be detected and there is no significant influence of the waiting time.

Thus, according to the d.s.c. experiments no crystallization or local ordering has been demonstrated in the
Table 2 Properties of polysulfone membranes. The data have been taken from ref. 14 (pervaporation) and from an internal report (ultrafiltration)

\begin{tabular}{lllllll}
\hline No. & Solvent & $\% \mathrm{PSf}$ & $\begin{array}{l}\text { Non- } \\
\text { solvent }\end{array}$ & $\begin{array}{l}J_{\mathrm{H}_{2} \mathrm{O}^{a}} \\
\left(\mathrm{~cm} \mathrm{hr}^{-1}\right)\end{array}$ & $\begin{array}{l}J^{b} \\
\left(10^{-2}\right. \\
\left.\mathrm{cm}^{-1}\right)\end{array}$ & $\alpha_{\mathrm{H}_{2} \mathrm{O}^{c}}$ \\
\hline I & DMAc & 15 & water & $18(3 \mathrm{~atm})$ & $--^{d}$ & - \\
II & DMAc & 35 & $\begin{array}{l}\text { water } \\
\text { III }\end{array}$ & $0(40 \mathrm{~atm})$ & 1.5 & 3.0 \\
IV & $\mathrm{CHCl}_{3}$ & 15 & $\begin{array}{l}\text { ethanol } \\
\text { ethanol }\end{array}$ & & 7.0 & 1.0 \\
\hline
\end{tabular}

a Pure water flux, pressure indicated is trans membrane difference

bervaporation permeate flux, feed: $50 \%$ water $/ 50 \%$ ethanol

c Selectivity for water

d Permeability is too high; no proper vacuum is attained

concentrated PSf solutions. To our knowledge no evidence of crystallinity has been reported in the literature for the class of the polysulfones, with the exception of solventinduced crystallization of poly(ethersulphone) ${ }^{15}$. Nevertheless, in a certain concentration range there must be a solution-gel transition since there do exist PSf films and membranes. Apparently in PSf solutions this gelation is not induced by crystalline regions. The possibility of amorphous local ordering, as proposed by Tan et al. , $^{9,10}$ will not be addressed here since this concept is not yet described in physical terms. Another possibility is to view the solution-gel boundary as a viscosity boundary. In very concentrated solutions the overlap between the polymer molecules is large and the entanglements are numerous. This drastically reduces the fluidity of the solution and as disentanglement will be slow, the system has viscoelastic properties. In this case the solution-gel transition is not a thermodynamic equilibrium and one does not expect a sharp, thermoreversible, gel point.

Preparation and properties of polysulfone membranes

The most important result in relation to membrane formation is the observation that gelation in the PSf system is not accompanied by any large scale ordering. This means that the molecules in gelled PSf film are evenly distributed and that transport through such a layer must take place via molecular diffusion using the free volume of the PSf molecules. Thus, in asymmetric PSf membranes with a skin layer formed by gelation the transport mechanism will be the solution-diffusion mechanism.

In Table 2 the characteristic properties of some polysulfone membranes are collected. Coagulation of a $15 \% \mathrm{PSf}-$ DMAc solution in water yields a typical ultrafiltration membrane with a significant permeability for water. Considering the hydrophobic nature of the polymer, we assume that in the skin layer there must be pores present through which a convective flow is possible. This is supported by the pervaporation experiments: the permeability is so high that no vacuum on the permeate side of the membrane is attained. If the PSf concentration in the casting solution is increased to $35 \%$, a pressure driven transport of water through the membrane is not possible. In pervaporation experiments this membrane shows a significant flu $x$ and a moderate selectivity for water. In our opinion this points to transport by diffusion and certainly not by convection. There are two reasons why in this case the filtration water flux is zero and the pervaporation flux is not: (i) a concentration gradient represents a much larger gradient in chemical potential (the true driving force) compared to a pressure gradient, and (ii) the water 
uptake by the polysulfone is facilitated by the presence of the ethanol ${ }^{13}$.

The difference in structure of the skin layer of the membranes I and II (see Table 2) can be explained with the ternary phase diagram given in Figure 12. In the skin layer of membrane II no pores are present, so the coagulation path responsible for the skin formation does not cross the binodal. Lowering of the PSf concentration in the casting solution increases the possibility of liquid-liquid demixing in the skin layer, if we assume that the direction of the coagulation path is unchanged. The coagulation path for the skin layer of membrane I crosses the binodal at a very high polymer concentration and this leads to pores with very small radii.

From the analysis given above it is clear that the location of the binodal is important with respect to the formation of the skin layer. In the polysulfone systems discussed here the binodal is determined mainly by the polymer-nonsolvent interaction. Nevertheless, the choice of the solvent remains crucial. The nature of the solvent may influence (i) the ratio of the solvent outflow and of the nonsolvent inflow, i.e. the direction of the coagulation path, and (ii) the structure of the formed gel. This is illustrated by the two last columns of Table 2. The membrane forming systems III and IV have almost the same location of the binodal, see Figure 9, but the membranes III and IV clearly differ in properties, the skin of membrane IV obviously being much denser.

\section{CONCLUSIONS}

The experimentally determined binodals of polysulfone/solvent/nonsolvent systems are in qualitative agreement with the Flory-Huggins lattice theory for polymer so- lutions. No evidence for crystallization has been found in concentrated solutions of polysulfone, so gelation in these systems is considered to be amorphous.

Gelation is responsible for the formation of the skin of asymmetric polysulfone pervaporation membranes, whereas liquid-liquid demixing creates the pores in the skin of polysulfone ultrafiltration membranes.

\section{REFERENCES}

1 Altena, F. W. PhD Thesis, Twente University of Technology, Enschede, The Netherlands, 1982, Ch. 3 and 4

2 Broens, L., Altena, F. W., Smolders, C. A. and Koenhen, D. M. Desalination 1980, 32, 33

3 Koenhen, D. M., Mulder, M. H. V. and Smolders, C. A. J. Appl. Polym. Sci. 1977, 21, 199

4 Smolders, C. A. in 'Ultrafiltration Membranes and Applications' (Ed. A. R. Cooper), Plenum Press, New York, 1980

5 Wijmans, J. G. and Smolders, C. A., to be published in the NATO ASI Series, Reidel Publishing Company

6 Altena, F. W. and Smolders, C. A. Macromolecules 1982, 15, 1491

7 Flory, P. J. 'Principles of Polymer Chemistry', Cornell University Press, Ithaca, 1953

8 de Gennes, P.-G. 'Scaling Concepts in Polymer Physics', Cornell University Press, Ithaca, 1979

9 Tan, H. M., Moet, A., Hiltner, A. and Baer, E. Macromolecules $1983,16,28$

10 Tan, H. M., Chang, B. H., Baer, E. and Hiltner, A. Eur. Polym. J. $1983,19,1021$

11 van Emmerik, P. T. and Smolders, C. A. J. Polym. Sci. C 1972, 38, 73

12 Wijmans, J. G. and Smolders, C. A. Eur. Polym. J. 1983, 19, 1143

13 Mulder, M. H. V., Franken, A. C. M. and Smolders, C. A. J Membrane Sci. in press

14 Mulder, M. H. V., Oude Hendrikman, J., Wijmans, J. G. and Smolders, C. A., submitted to J. Appl. Polym. Sci.

15 Blackadder, D. A., Ghavamikia, H. and Windle, A. H. Polymer $1979,20,781$ 